\title{
Checklist of the cicadas (Insecta: Hemiptera: Cicadidae) of Nicaragua including new records for seventeen species
}

\author{
Allen F. Sanborn ${ }^{1 *}$ and Jean-Michel Maes ${ }^{2}$ \\ 1 Barry University, Department of Biology, 11300 NE Second Avenue, Miami Shores, FL 33161-6695, U.S.A. \\ 2 Museo Entomológico, AP 527, León, Nicaragua \\ * Corresponding author. E-mail: asanborn@mail.barry.edu
}

\begin{abstract}
A current checklist of the Nicaraguan cicada fauna is provided. The first Nicaraguan records of Miranha imbellis (Walker, 1858), Diceroprocta bicosta (Walker, 1850), Diceroprocta ruatana (Distant, 1891), Fidicina mannifera (Fabricius, 1803), Fidicinoides cachla (Distant, 1899), Fidicinoides pronoe (Walker, 1850), Fidicinoides spinicosta (Walker, 1850), Pacarina championi (Distant, 1881), Pacarina schumanni Distant, 1905, Majeorona aper (Walker, 1850), Majeorona truncata Goding, 1925, Quesada gigas (Olivier, 1790), Procollina n. sp., Carineta cinara Distant, 1883, Carineta postica Walker, 1858, Carineta viridicata Distant, 1883, Herrera ancilla (Stål, 1864), Calyria telifera (Walker, 1858) and Conibosa occidentis (Walker, 1858) are provided. The new records increase the known cicada diversity $380 \%$ bringing the total number of cicada species reported in Nicaragua to 24 species in 16 genera representing eight tribes and two subfamilies of the family Cicadidae.
\end{abstract}

\section{INTRODUCTION}

Cicadas are large and noisy insects that are known for the calling songs they produce. Although generally obvious in their environment, they are highly mobile, found at low population densities, difficult to catch and rarely cause agricultural damage. As a result, cicadas are generally taken as part of larger, non-specific collection efforts and often remain undetermined in various museum collections for extended periods of time and unrepresented in the fauna of particular countries. Several papers describing the cicada fauna, providing new records or describing new species of cicadas in various countries in the New World have been published in the last decade (Boulard and Martinelli 2011; Maccagnan et al. 2011; Sanborn 2001a, b; 2004; 2005; 2006a, b; 2007a, b; 2008; 2009; 2010a, b; 2011a, b, c; Santos and Martinelli 2009a; 2009b; Santos et al. 2010; Thouvenot 2007). This series of papers has been produced to provide information on the regional fauna while expanding the information of biogeography of individual cicada species and to assist with further species determinations. This work is a continuation of these cicada studies and the work of J.M.M. in producing an inventory of Nicaraguan insects. We describe new records for Nicaragua along with the first listing of species known for the country.

\section{Materials AND Methods}

Specimens of 17 species representing new records for Nicaragua were found among the undetermined cicadas at the Museo Entomológico de León (MEL-LEON) or were collected by J.M.M. All specimens were collected with permits issued by the Environmental Ministry of Nicaragua (MARENA). Voucher specimens for the new records are deposited in the MEL-LEON and Sanborn collections (AFSC) as listed with each species. Records for other species inhabiting Nicaragua were determined by searching the cicada bibliographies (Metcalf 1963a, b, c;
Duffels and van der Laan 1985) and individual references can be found there. Data for geographical distribution of the species were collected from the cicada bibliographies and more recent literature (Wolda and Ramos 1992; Sanborn 2001a; 2006a, b; 2007a, b; 2010a, b; 2011a, b).

\section{RESULTS AND DiscusSion}

The cicada bibliographies provided reference to only four species inhabiting Nicaragua. One additional record has been published recently (Sanborn 2010b). New records for 18 additional species were found in the undetermined material of the MEL-LEON.

\section{List of new species}

Miranha imbellis (Walker, 1858) (Subfamily Cicadinae, Tribe Zammarini)

Geographical distribution. Miranha imbellis has previously been reported from Guatemala, Honduras, Mexico and Panama (Metcalf 1963a; Wolda and Ramos 1992; Sanborn 2006a; 2007b).

Specimen examined. NICARAGUA, Zelaya, Cerro Saslaya, Camp 3, 950 m, 1 - IV - 1999, UTM-713150/1521450, col. Jean-Michel Maes and B. Hernández, MEL/MAES 446301446302 (2 males, MEL-LEON); ibidem, 1 - IV - 1996, col. Jean-Michel Maes, MEL/MAES 446303 (1 male, MELLEON).

Diceroprocta bicosta (Walker, 1850) (Subfamily Cicadinae, Tribe Cryptotympanini, Subtribe Cryptotympanaria)

Geographical distribution. Diceroprocta bicosta has previously been reported from Colombia, Costa Rica, El Salvador, Honduras and Mexico (Metcalf 1963a; Duffels and van der Laan 1985; Sanborn 2001a; 2006a; 2007a; 2010a).

Specimen examined. NICARAGUA, Managua, Mateare, 29 - V - 1995, S, Jatropha curcas, col. C. Grimm, MEL/ 
MAES 446082 (1 male, MEL-LEON); León, 1 - VIII - 1988, B. Garcete, MEL/MAES 446090 (1 female, MEL-LEON); ibidem, R. Oulton, MEL/MAES 446089 (1 female, MELLEON); ibidem, T. Jovel, MEL/MAES 446087 (1 female, MEL-LEON); ibidem, Jean-Michel Maes, MEL/MAES 446086 (1 female, MEL-LEON); León, Rio Pochote, 1 - V 1997, B. Garcete, MEL/MAES 446084 (1 male, MEL-LEON); ibidem, T. Jovel, MEL/MAES 446083 (1 male, MEL-LEON); León, Volcán Telica, 1 - VII - 1990, Jean-Michel Maes, MEL/MAES 446080 (1 male, MEL-LEON); Chinandea, Chichigalpa, Ingenio San Antonio, 1 - I - 2001, B. Garcete, MEL/MAES 446081 (1 male, MEL-LEON); Granada, Las

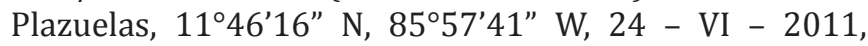
Jean-Michel Maes, MEL/MAES 53310 (1 female, MELLEON); Rivas Dept., Cárdenas, Finca Isla Vista, 11¹3'47” N, 85³3'04" W", 8 - VII - 2010, Jean-Michel Maes, MEL/ MAES 53432-53433 (2 females, MEL-LEON).

Diceroprocta ruatana (Distant, 1891) (Subfamily Cicadinae, Tribe Cryptotympanini, Subtribe Cryptotympanaria)

Geographical distribution. Diceroprocta ruatana has previously been reported from Guatemala and Honduras (Metcalf 1963a; Sanborn 2006a; 2010b).

Specimens examined. NICARAGUA, Managua, Ticuantepe, Montibelli, 5/10 - V - 2003, col. D. Roíz, MEL/ MAES 446103-446104 (2 males, MEL-LEON); Granada, Domitila, alt 55m, 1 - V - 2010, col. Jean-Michel Maes, MEL/MAES 53311-53313 (3 males, MEL-LEON); León, V 90, B. Garcete, MEL/MAES 446091 (1 female, MEL-LEON); ibidem, 15 - V - 1984, col. T. Anton, MEL/MAES 446085 (1 female, MEL-LEON); ibidem, 1 - V - 1987, col. T. Anton, MEL/MAES 446088 (1 female, MEL-LEON).

Fidicina mannifera (Fabricius, 1803) (Subfamily Cicadinae, Tribe Fidicinini, Subtribe Fidicinina)

Geographical distribution. The species has been reported previously from the Antilles, Bolivia, Brazil, Colombia, Costa Rica, Ecuador, French Guiana, Guyana, Panama, Paraguay, Peru and Surinam (Metcalf 1963a; Duffels and van der Laan 1985; Boulard and Martinelli 1996; Wolda and Ramos 1992; Sanborn 2010a; 2011b).

Specimens examined. NICARAGUA, Zelaya, Cerro Saslaya, 850 m (bosque), 7/14 - VII - 99, col. B. Hernández, MEL/MAES 445948 (1 female, MEL-LEON); Region Autonoma Atlantico Norte, Cerro Cola Blanca: Rio Las Latas, $14^{\circ} 04^{\prime} 12^{\prime \prime} \mathrm{N}, 8^{\circ} 32^{\prime} 44^{\prime \prime} \mathrm{W}, 2$ - VI - 1997, col. JeanMichel Maes, MEL/MAES 445947 (1 male, MEL-LEON).

Fidicinoides cachla (Distant, 1899) (Subfamily Cicadinae, Tribe Fidicinini, Subtribe Fidicinina)

Geographical distribution. Fidicinoides cachla has previously been reported from Costa Rica and Honduras (Metcalf 1963a; Sanborn 2006a).

Specimens examined. NICARAGUA, Zelaya, $8 \mathrm{~km} \mathrm{E}$ Bonanza, CICABO 1 - IV - 2000, col. Jean-Michel Maes and B. Hernández, MEL/MAES 446059-446066, 446105$446117, \quad 446119-446132,446134-446140, \quad 446143-$ 446145-446169, 446171-446173 (38 males, 36 females, MEL-LEON); ibidem, MEL/MAES 446118 (1 male, AFSC); ibidem, MEL/MAES 446170 (1 female, AFSC); Region Autonoma Atlantico Norte, Rio Coco, Amaca, 14¹6'51" N, 8509'54" W, 9 - IV - 2002, Col. Jean-Michel Maes, MEL/
MAES 466055-446057 ( 3 females, MEL-LEON); Zelaya, Rio Las Latas, $14^{\circ} 04^{\prime} 00^{\prime \prime} \mathrm{N}, 84^{\circ} 33^{\prime} 00^{\prime \prime} \mathrm{W}, 2$-VI-1997,220 m, Col. Jean-Michel Maes and B. Hernández, MEL/MAES 446058 (1 female, MEL-LEON); Region Autonoma Atlantico Norte, Siuna, El Hormiguero, 128 m, 13²5'21' N, 8457'00” W, 25 - II - 2004, Col. Jean-Michel Maes, MEL/MAES 466174 (1 female, MEL-LEON); Zelaya: Cerro Saslaya: Camp 2: $700 \mathrm{~m}$ IV - 1999, UTM-714750/1518900, Col. Jean-Michel Maes and B. Hernández, MEL/MAES 446175-446182, 446187445988 (10 females, MEL-LEON); ibidem, 1 - IV - 1996, MEL/MAES 446179, 446183-446186, 446188 (6 females, MEL-LEON); Granada, Volcán Mombacho, Finca Santa Ana 3, trampa malaise en café bajo cultivo orgánica, alt., 600, m, 1150'24" N, 8559'56" W, 31 - III - 1998, col. JeanMichel Maes, MEL/MAES 446190 (1 female, MEL-LEON); ibidem, 16 - II - 1998, MEL/MAES 446189 (1 female, MELLEON); ibidem, 18 - III - 1999, MEL/MAES 446191 (1 female, MEL-LEON); Granada, Volcán Mombacho, Bosque

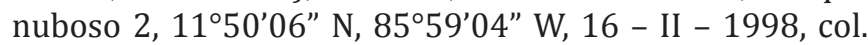
Jean-Michel Maes, MEL/MAES 446192 (1 female, MELLEON); Granada, Volcán Mombacho, Finca El Progreso 1, trampa malaise en café bajo cultivo quìmico, $11^{\circ} 50^{\prime} 05^{\prime \prime} \mathrm{N}$, 8559'40" W, 31 - III - 1998, col. Jean-Michel Maes, MEL/ MAES 446193, 446197 (2 females, MEL-LEON); Granada, Volcán Mombacho, Finca San Joaquin 1, trampa malaise en

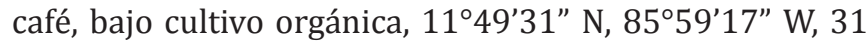
- III - 1998, col. Jean-Michel Maes, MEL/MAES 446194 (1 female, MEL-LEON); ibidem, 30 - IV - 1998, MEL/MAES 446196, 446198 (2 females, MEL-LEON); Granada, Volcán Mombacho: Finca San Joaquin 2, trampa malaise en café bajo cultivo orgánica, 1149’31” N, 8559'17” W, 18 - III 1999, col. Jean-Michel Maes, MEL/MAES 446195 (1 female, MEL-LEON); Rìo San Juan, El Castillo de la Concepcion,

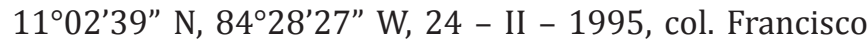
Collantes, 446199-446200 (2 females, MEL-LEON); Rivas Dept., Cárdenas, Finca Guadalupe, 28 - II/1 - III - 2009, col. Jean-Michel Maes (51 males, 14 females, AFSC).

Fidicinoides pronoe (Walker, 1850) (Subfamily Cicadinae, Tribe Fidicinini, Subtribe Fidicinina)

Geographical distribution. Fidicinoides pronoe has previously been reported from Brazil, Colombia, Costa Rica, Ecuador, El Salvador, Guatemala, Honduras, Mexico, Panama, Peru, Trinidad and Tobago and Venezuela (Metcalf 1963a; Duffels and van der Laan 1985; Wolda and Ramos 1992; Sanborn 2001a; 2006a; 2007a, b; 2010a).

Specimens examined. NICARAGUA, Jinotega, Santa Enriqueta, 1250 m, 29 - I - 4 - IV -2003, col. D. Roíz, MEL/ MAES 446072-446073 (2 males, MEL-LEON); Zelaya, Rio Las Latas, $14^{\circ} 04^{\prime} 00^{\prime \prime} \mathrm{N}, 8^{\circ} 33^{\prime} 00^{\prime \prime} \mathrm{W}, 2$ - VI - 1997, 220 m, col. Jean-Michel Maes and B. Hernández, MEL/MAES 446074-466075 (2 females, MEL-LEON); Jinotega, Reserva Natural Danatli, El Diablo, 1 - IV - 1997, col. Jean-Michel Maes, MEL/MAES 445895-445896 (2 males, MEL-LEON).

Fidicinoides spinicosta (Walker, 1850) (Subfamily Cicadinae, Tribe Fidicinini, Subtribe Fidicinina)

Geographical distribution. Fidicinoides spinicosta has previously been reported from Brazil, Colombia, Costa Rica and Panama (Metcalf 1963a; Duffels and van der Laan 1985; Sanborn 2010a).

Specimen examined. NICARAGUA, Rio San Juan, 
Refugio Bartola, 10 - XI - 1999, Jean-Michel Maes and B. Hernández, MEL/MAES 446069 (1 male, MEL-LEON); ibidem, 1 - VI - 2005, MEL/MAES 445900 (1 female, MEL-LEON); ibidem, 20 - VI - 2004, MEL/MAES 446068 (1 male, MEL-LEON); ibidem, 3 - VI - 2003, MEL/MAES 446070 ( 1 male, MEL-LEON).

Pacarina championi (Distant, 1881) (Subfamily Cicadinae, Tribe Fidicinini, Subtribe Guyalnina)

Geographical distribution. The species has previously been reported from Belize, Costa Rica, El Salvador, Guatemala, Honduras, Mexico and Panama (Metcalf 1963a; Wolda and Ramos 1992; Sanborn 2001a; 2006a; 2007b).

Specimens examined. NICARAGUA, León, VIII - 1990, B. Garcete, MEL/MAES 446037 (1 male, MEL-LEON); ibidem, 1 - VII - 1989, Jean-Michel Maes, MEL/MAES 446038 (1 female, MEL-LEON); ibidem, 1 - VIII - 1990, MEL/MAES 446039 (1 female, MEL-LEON); ibidem, 1 - VI - 1995, MEL/MAES 446041 (1 female, MEL-LEON); ibidem, 1 VI - 1990, MEL/MAES 446042 (1 female, MEL-LEON); León, Cerro Negro, 20 - VIII - 1989, Blas Hernández, MEL/ MAES 446034 (1 male, MEL-LEON); ibidem, 20 - II - 1986, B. Garcette, MEL/MAES 446035 (1 female, MEL-LEON); Masaya, Las Flores, VI - 1993, J. Tellez and Jean-Michel Maes, MEL/MAES 446022 (1 female, MEL-LEON); León, CIMAC, 24 - VI - 2000, trampa luminica, col. M. Torres and B. Hernandez, MEL/MAES 446040 (1 female, MELLEON); ibidem, Jean-Michel Maes, MEL/MAES 446044 (1 female, MEL-LEON); Managua, Laguna de Xiloa, 20 - I - 1994, B. Garcete, MEL/MAES 446047 (1 female, MELLEON); Masaya, Las Flores, 1 - VI - 1994, B. Garcete, MEL/ MAES 446048 ( 1 female, MEL-LEON); Carazo, La Trinidad, 11'44'13" N, 86¹9'59" W, 10 - XII - 2004, Jean-Michel Maes, MEL/MAES 446050 (1 female, MEL-LEON); Carazo, Chococente, 11 - IX - 1992, F. Reinholdt, MEL/MAES 446051 (1 female, MEL-LEON).

Pacarina schumanni Distant, 1905 (Subfamily Cicadinae, Tribe Fidicinini, Subtribe Guyalnina)

Geographical distribution. Pacarina schumanni has previously been reported from Belize, El Salvador, Guatemala, Honduras and Mexico (Metcalf 1963a; Sanborn 2001a; 2006a; 2007b; 2010b; Sueur 2002).

Specimen examined. NICARAGUA, Granada, Domitila, alt 55m, UTM-16P-0614035-1294527, 27 - V/1 - VI 2002, col. Jean-Michel Maes, MEL/MAES 446052, 445901, 53351 ( 2 females, 1 male, MEL-LEON); ibidem, col. P. Schmidt, MEL/MAES 446053 (1 female, MEL-LEON); Rio San Juan, Indio Maiz - camp 2, 1059'22” N, 84¹1'01” W, 8 - VI - 2002, col. P. Schmidt and B. Hernandez, MEL/MAES 445997 (1 female, MEL-LEON).

Majeorona aper (Walker, 1850) (Subfamily Cicadinae, Tribe Fidicinini, Subtribe Guyalnina)

Geographical distribution. The species has previously been reported from Brazil, Colombia, Ecuador, French Guiana and Panama (Metcalf 1963a; Wolda and Ramos 1992; Sanborn 2010a; 2011b).

Specimens examined. NICARAGUA, Zelaya, Rio Las Latas, $14^{\circ} 04^{\prime} 00^{\prime \prime} \mathrm{N}, 8^{\circ} 33^{\prime} 00^{\prime \prime} \mathrm{W}, 2$ - VI - 97, 220 m, col. Jean-Michel Maes and B. Hernández, MEL/MAES 445940445941, 445943-445946 (6 females, MEL-LEON); ibidem
MEL/MAES 445942 (1 female, AFSC).

Majeorona truncata Goding, 1925 (Subfamily Cicadinae, Tribe Fidicinini, Subtribe Guyalnina)

Geographical distribution. The species has previously been reported from Brazil, Ecuador and Honduras (Metcalf 1963a; Sanborn 2006a; 2008; 2010b).

Specimens examined. NICARAGUA, Zelaya, Rio Las Latas, $14^{\circ} 04^{\prime} 00^{\prime \prime} \mathrm{N}, 84^{\circ} 33^{\prime} 00^{\prime \prime} \mathrm{W}, 2$ - VI - 97, $220 \mathrm{~m}$, col. Jean-Michel Maes and B. Hernández, MEL/MAES 446071 (1 female, MEL-LEON).

Quesada gigas (Olivier, 1790) (Subfamily Cicadinae, Tribe Hyantiini)

Geographical distribution. The species has previously been reported from the Antilles, Argentina, Belize, Bolivia, Brazil, Colombia, Costa Rica, Ecuador, El Salvador, French Guiana, Guatemala, Guyana, Honduras, Mexico, Panama, Paraguay, Peru, Trinidad and Tobago, Uruguay, United States, Venezuela and the West Indies (Metcalf 1963a; Duffels and van der Laan 1985; Wolda and Ramos 1992; Sanborn 2001a; 2006b; 2007a, b; 2010a; 2011a, b; Sueur, 2002).

Specimens examined. NICARAGUA, Zelaya, $8 \mathrm{~km} \mathrm{E}$

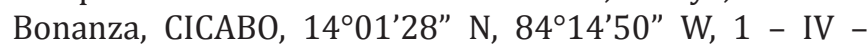
2000, col. Jean-Michel Maes and B. Hernández, MEL/MAES 446201-446232, 446234-446259 (58 males MEL-LEON); ibidem, MEL/MAES 446233 (1 male AFSC); ibidem, 16 - V2000, col. Juan Pablo Orozco, MEL/MAES 445935-445937 (3 females, MEL-LEON); Granada, Volcan Mombacho, Santa Ana \#2, 31 - III - 1998, col. Jean-Michel Maes, MEL/ MAES 446264 (1 male, MEL-LEON); ibidem, 1 - VIII 2002, MEL/MAES 446265 (1 male, MEL-LEON); Region Autonoma Atlantico Norte, Siuna, El Hormiguero, 128 m, $13^{\circ} 45^{\prime} 21^{\prime \prime} \mathrm{N}, 84^{\circ} 57^{\prime} 00^{\prime \prime} \mathrm{W}, 25$ - II - 2004, col. Jean-Michel Maes, MEL/MAES 460269 (1 male, MEL-LEON); Leon, Leon, 12²6'09” N, 8652'56" W, 1 - VIII - 1992, col. JeanMichel Maes, MEL/MAES 446261 (1 male, MEL-LEON); Boaco, Camoapa: Santa Elena, 12²4'47” N, 8527'43” W, 18 - IV - 2003, col. Jean-Michel Maes, MEL/MAES 446262 (1 male, MEL-LEON); Masaya, Catarina, 11 ${ }^{\circ} 54^{\prime} 43^{\prime \prime} \mathrm{N}$, 8604'29” W, 1 - II - 1988, col. Jean-Michel Maes, MEL/ MAES 446263 (1 male, MEL-LEON); Region Autonoma Atlantico Sur, Nueva Guinea: Monte Verde: Finca Dona

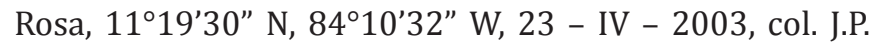
Desmedt, MEL/MAES 446266 (1 male, MEL-LEON).

Procollina n. sp. (Subfamily Cicadettinae, Tribe Dazini)

Geographical distribution. This new species is only known from Nicaragua (Sanborn in preparation).

Specimens examined. NICARAGUA, Jinotega Department, Cerro Kilambé, Camp 2 Los Quetzales, UTM 16P-1503500-0638900, 1400 m, 23/30 - IV - 2001, col. J. Sunyar and B. Hernandez, MEL/MAES 446307-446309 (3 females, MEL-LEON); ibidem, Camp 1, UTM 16P1503500-0638900, MEL/MAES 446304-446306 (1 male, 2 females, MEL-LEON); ibidem, Camp 6 Las Torres, UTM 16P-1500100-0637100, 1400 m, MEL/MAES 46310 (1 male, MEL-LEON); Jinotega Department, Penias Blancas, $13^{\circ} 17^{\prime} 00^{\prime \prime} \mathrm{N}, 85^{\circ} 38^{\prime} 00^{\prime \prime} \mathrm{W}, 25$ - VII - 1997, col. Jean-Michel Maes and B. Hernandez, MEL/MAES 446311-446312 (2 females, MEL-LEON); Jinotega Department, El Diablo, 
Datanli, $13^{\circ} 06^{\prime} 20^{\prime \prime} \mathrm{N}, 8^{\circ} 51^{\prime} 28^{\prime \prime} \mathrm{W}$, bosque nebulina, 1483 m, IV - 2005, col. Jean-Michel Maes, MEL/MAES 445877 (1 female, MEL-LEON).

Carineta cinara Distant, 1883 (Subfamily Cicadettinae, Tribe Carinetini)

Geographical distribution. Carineta cinara has previously been reported from Costa Rica, Guatemala and Panama (Metcalf 1963c; Wolda and Ramos 1992; Sanborn 2010b).

Specimens examined. NICARAGUA, Region Autonoma Atlantico Norte, Cerro Cola Blanca: Rio Las Latas, $14^{\circ} 04^{\prime} 12^{\prime \prime} \mathrm{N}, 84^{\circ} 32^{\prime} 44^{\prime \prime}$ W, 2 - VI - 1997, col. Jean-Michel Maes, MEL/MAES 445991-445995 (1 male, 4 females, MEL-LEON); ibidem, P. Schmit, MEL/MAES 445990 (1 male, MEL-LEON); Rio San Juan, Indio Maiz - camp 2, $10^{\circ} 59^{\prime} 22^{\prime \prime}$ N, 8411'01" W, 8/10 - VI - 2002, col. P. Schmidt and B. Hernandez, MEL/MAES 445997 (1 female, MELLEON); ibidem, col. Jean-Michel Maes, MEL/MAES 445996 (1 female, MEL-LEON).

Carineta postica Walker, 1858 (Subfamily Cicadettinae, Tribe Carinetini)

Geographical distribution. Carineta postica has previously been reported from Colombia, Costa Rica, Ecuador, Guatemala Panama, and Peru (Metcalf 1963c; Wolda and Ramos 1992; Sanborn 2010b).

Specimens examined. NICARAGUA, Jinotega: El Jaguar - alt. 1346 m, UTM-16P-06002629-1462653, 1314' N, 8409' W, 12 - VIII - 2010, col. Jean-Michel Maes, MELLEON 53314 (1 male, MEL-LEON).

Carineta viridicata Distant, 1883 (Subfamily Cicadettinae, Tribe Carinetini)

Geographical distribution. Carineta viridicata has previously been reported from Bolivia, Colombia, Panama and Peru (Metcalf 1963c; Wolda and Ramos 1992; Sanborn 2010a).

Specimen examined. NICARAGUA,Zelaya: Cerro Saslaya: Camp 2: 700 m - IV - 1999, UTM-714750/1518900, col. Jean-Michel Maes and B. Hernández, MEL/MAES 445984445989 ( 5 males, 1 female, MEL-LEON).

Herrera ancilla (Stål, 1864) (Subfamily Cicadettinae, Tribe Carinetini)

Geographical distribution. Herrera ancilla has previously been reported from Belize, El Salvador, Guatemala, Honduras, Mexico and Panama (Metcalf 1963c; Wolda and Ramos 1992; Sanborn 2001a; 2006a; 2007b).

Specimens examined. NICARAGUA, Rio San Juan: Refugio Bartola, 1 - V - 2010, 40 m, 1058'23" N, 84²0'19" W, col. Jean-Michel Maes, MEL-LEON 53325-53337 (6 males, 7 females, MEL-LEON); ibidem, 1 - VI - 2005, MELLEON 53323 (1 female, MEL-LEON), ibidem, MEL-LEON 53324 (1 male, MEL-LEON); ibidem, VI - 2008, 1058'23" N, 8420'19" W (1 male, AFSC); Matagalpa: Selva Negra: 1300 m, 1 - V - 2010, col. Jean-Michel Maes, MEL-LEON 445913, 445998-445999, 533315-533321 (1 male, 9 females, MEL-LEON); ibidem, 1 - VII - 2005, MEL-LEON 53322 (1 male, MEL-LEON); Region Autonoma Atlantico Norte, Cerro Cola Blanca: Rio Las Latas, $14^{\circ} 04^{\prime} 12^{\prime \prime} \mathrm{N}$, 84³2'44" W, 2 - VI - 1997, col. Jean-Michel Maes, MEL/
MAES 445911-445912 (2 males, MEL-LEON).

Calyria telifera (Walker, 1858) (Subfamily Cicadettinae, Tribe Parnisini)

Geographical distribution. Calyria telifera has previously been reported from Brazil, Costa Rica, Honduras, Peru and Venezuela (Metcalf 1963c; Sanborn 2007a; 2010b).

Specimen examined. NICARAGUA, Rio San Juan: Refugio Bartola, 1058'20” N, 84²0'20” W, 20/24 - VI 2004, col. Jean-Michel Maes, MEL/MAES 445908 ( 1 female, MEL-LEON); ibidem, 1 - VI - 2005, MEL/MAES 445905445906 (2 females, MEL-LEON); ibidem, 3 - VI - 2003, MEL/MAES 445907 (1 female, MEL-LEON); ibidem, VI 2008, 40 m, 1058'23” N, 84²0'19” W (1 female, AFSC); Region Autonoma Atlantico Norte, Cerro Cola Blanca: Rio Las Latas, 14.07N, 84.54555W, 2 - VI - 1997, col. JeanMichel Maes, MEL/MAES 445902-445904 (2 males, 1 female, MEL-LEON).

Conibosa occidentalis (Walker, 1858) (Subfamily Cicadettinae, Tribe Chlorocystini)

Geographical distribution. Conibosa occidentalis has previously been reported from Honduras, Mexico and Panama (Metcalf 1963c; Wolda and Ramos 1992; Sanborn 2007b; 2010b).

Specimen examined. NICARAGUA, Rio San Juan: Refugio Bartola, 6/9 - II - 2000, col. Jean-Michel Maes and B. Hernandez, MEL/MAES 445909 (1 male, MEL-LEON); ibidem, 1 - V - 2000, MEL/MAES 445910 (1 female, MELLEON); ibidem, 1 - V - 2010, MEL/MAES 53352 (1 female, MEL-LEON).

The new records bring the reported cicada fauna of Nicaragua to 24 species in 16 genera from eight tribes and two subfamilies of the family Cicadidae. The current list of species and their taxonomic position is provided in Table 1. Some of these records filled in a gap in the given species distribution joining previous records from north and south of Nicaragua, e.g. Quesada gigas. Others represent a southern (e.g. Pacarina schumanni) or northern expansion (e.g. Fidicina mannifera) of the known range of a species. Of the five species previously reported from Nicaragua, Odopoea diriangani (Distant, 1881), Proarna insignis Distant, 1881 and Ollanta modesta (Distant, 1881) were originally described with specimens from Nicaragua and Zammara smaragdina Walker, 1850 has been reported from Nicaragua for an extended time (Distant 1881), while P. sallaei Stål, 1864 was only recently recorded as part of the Nicaraguan cicada fauna (Sanborn 2010b).

There are still multiple species that will probably be captured in Nicaragua. There are currently 28 species in the Honduran and 31 species in the Costa Rica cicada fauna, 12 and 16 of which have not been reported from Nicaragua respectively, and nine species that have been reported from countries both north and south of Nicaragua (Metcalf 1963a, b, c; Duffels and van der Laan 1985; Sanborn 2005; 2006a; 2010b; Sanborn et al. 2008). The significant increase in the known fauna with this work illustrates what a little concentrated effort can achieve in determining and adding to the known fauna for a region. 
TABLE 1. Taxonomic list of cicadas currently reported to inhabit Nicaragua.

\begin{tabular}{l}
\hline Family Cicadidae Leach, 1815 \\
\hline Subfamily Cicadinae Latrielle, 1802 \\
Tribe Zammarini Distant, 1905 \\
Odopoea Stål, 1861 \\
Odopoea diriangani (Distant, 1881) \\
Miranha Distant, 1905 \\
Miranha imbellis (Walker, 1858) \\
Zammara Amyot and Audinet-Serville, 1843 \\
Zammara smaragdina Walker, 1850 \\
Tribe Cryptotympanini Handlirsch, 1925 \\
Sub-tribe Cryptotympanaria Handlirsch, 1925 \\
Diceroprocta Stål, 1870 \\
Diceroprocta bicosta (Walker, 1850) \\
Diceroprocta ruatana (Distant, 1891) \\
Tribe Fidicinini Distant, 1905 \\
Sub-tribe Fidicinina Distant, 1905 \\
Fidicina Amyot and Audinet-Serville, 1843 \\
Fidicina mannifera (Fabricius, 1803) \\
Fidicinoides Boulard and Martinelli, 1996 \\
Fidicinoides cachla (Distant, 1899) \\
Fidicinoides pronoe (Walker, 1850) \\
Fidicinoides spinicosta (Walker, 1850)
\end{tabular}

\section{Sub-tribe Guyalnina Boulard and Martinelli, 1996}

Proarna Stål, 1864

Proarna insignis Distant, 1881

Proarna sallaei Stål, 1864

Ollanta Distant, 1905

Ollanta modesta (Distant, 1881)

Pacarina Distant, 1905

Pacarina championi (Distant, 1881)

Pacarina schumanni Distant, 1905

Majeorona Distant, 1905

Majeorona aper (Walker, 1850)

Tribe Hyantiini Distant, 1905

Quesada Distant, 1905

Quesada gigas (Olivier, 1790)

\section{Subfamily Cicadettinae Buckton, 1889}

Tribe Dazini Kato, 1932

Procollina Metcalf, 1963

Procollina n. sp.

\section{Tribe Carinetini Distant, 1905}

Carineta Amyot and Audinet-Serville, 1843

Carineta cinara Distant, 1883

Carineta viridicata Distant, 1883

Herrera Distant, 1905

Herrera ancilla (Stål, 1864)

\section{Tribe Parnisini Distant, 1905}

Calyria Stål, 1862

Calyria telifera (Walker, 1858)

\section{Tribe Chlorocystini Distant, 1905}

Conibosa Distant, 1905

Conibosa occidentis (Walker, 1858)

ACKNowledgments: Reviewers Douglas Henrique Bottura Maccagnan and Rodrigo Souza Santos made suggestions to improve the manuscript.

\section{Literature Cited}

Boulard, M. and N.M. Martinelli. 1996. Révision des Fidicini, nouveau statut de la tribu, espèces connues et nouvelles espèces (Cicadomorpha, Cicadidae, Cicadinae). Premiere partie: Sous-tribu nouvelle des Fidicinina. Ecole Pratique des Hautes Etudes, Travaux du Laboratoire Biologie et Evolution des Insectes Hemipteroidea 9: 11-81.
Boulard, M. and N.M. Martinelli. 2011. Nouvelles cigales néotropicales de las sous-tribu des Guyalnina (Rhynchota, Cicadidae, Cicadinae, Fidicinini). Lambillionea 111: 219-232.

Distant, W.L. 1881. Rhynchota: Homoptera. Biologia Centrali-Americana; contributions to the knowledge of the fauna and flora of Mexico and Central America. Part 15, 1: 1-16.

Duffels, J.P. and P.A. van der Laan. 1985. Catalogue of the Cicadoidea (Homoptera, Auchenorhyncha) 1956-1980. Dordrect: Dr. W. Junk Publishers. 414 p. (Series Entomologica: 34).

Maccagnan, D.H.B., N.M. Martinelli, G. Goemans and E. Guilherme. 2011. Zammara smaragdula Walker, 1858 (Hemiptera: Cicadidae): first record for Brazil. Check List 7: 563-564.

Metcalf, Z.P. 1963a. General catalogue of the Homoptera, Fascicle VIII. Cicadoidea. Part 1. Cicadidae. Section I. Tibiceninae. North Carolina State College Contribution 1502: i-vii, 1-585.

Metcalf, Z.P. 1963b. General catalogue of the Homoptera, Fascicle VIII. Cicadoidea. Part 1. Cicadidae. Section II. Gaeninae and Cicadinae. North Carolina State College Contribution 1502: 587-919.

Metcalf, Z.P. 1963c. General catalogue of the Homoptera, Fascicle VIII. Cicadoidea. Part 2. Tibicinidae. North Carolina State College Contribution 1564: i-vi, 1-492.

Sanborn, A.F. 2001a. A first contribution to a knowledge of the cicada fauna of El Salvador (Homoptera: Cicadoidea). Florida Entomologist 84(3): 449-450.

Sanborn, A.F. 2001b. Distribution of the cicadas (Homoptera: Cicadidae) of the Bahamas. Florida Entomologist 84(4): 651-652.

Sanborn, A.F. 2004. Two new cicada Zammara species from South America (Hemiptera: Cicadoidea: Cicadidae). Florida Entomologist 87: $365-371$.

Sanborn, A.F. 2005. Fidicina variegata, a new cicada species from Costa Rica (Hemiptera: Cicadomorpha: Cicadidae). Annals of the Entomological Society of America 98: 187-190.

Sanborn, A.F. 2006a. New records for the cicada fauna from four Central American countries (Hemiptera: Cicadoidea: Cicadidae). Florida Entomologist 89(1): 75-79.

Sanborn, A.F. 2006b. New records of cicadas from Mexico (Hemiptera: Cicadoidea: Cicadidae). Southwestern Naturalist 51(2): 255-257.

Sanborn, A.F. 2007a. Additions to the cicada fauna of Venezuela with the description of a new species and checklist of the Venezuelan cicada fauna (Hemiptera: Cicadoidea: Cicadidae). Zootaxa 1503: 21-32.

Sanborn, A.F. 2007b. New species, new records and checklist of cicadas from Mexico (Hemiptera: Cicadoidea: Cicadidae). Zootaxa 1651: $1-42$

Sanborn, A.F. 2008. New records of Brazilian cicadas including the description of a new species (Hemiptera: Cicadoidea, Cicadidae). Neotropical Entomology 37(6): 685-690.

Sanborn, A.F. 2009. Checklist, new species and key to the cicadas of Cuba (Hemiptera: Cicadoidea: Cicadidae). Mitteilungen aus dem Museum für Naturkunde in Berlin - Deutsche Entomologische Zeitschrift 59(1): 85-92.

Sanborn, A.F. 2010a. The cicadas of Colombia including new records and the description of a new species (Hemiptera: Cicadidae). Journal of Natural History 44(25-28): 1577-1607.

Sanborn, A.F. 2010b. New records and new species of Central American cicadas (Hemiptera: Cicadoidea: Cicadidae). Studies on Neotropical Fauna and Environment 45(2): 67-76.

Sanborn, A.F. 2011a. Checklist of cicadas (Insecta: Hemiptera: Cicadidae) from Paraguay including new records for six species. Check List 7(4): 465-467.

Sanborn, A.F. 2011b. Checklist of the cicadas of French Guiana including new records and the description of nine new species (Insecta, Hemiptera, Cicadoidea, Cicadidae). Zoosystema 33(3): 377-418.

Sanborn, A.F. 2011c. A new species of Orellana Distant from Brazil (Hemiptera: Cicadoidea: Cicadidae). Proceedings of the Entomological Society of America 113: 377-384.

Sanborn, A.F., T.E. Moore and A.M. Young. 2008. Two new cicada species from Costa Rica (Hemiptera: Cicadomorpha: Cicadidae) with a key to Fidicinoides in Costa Rica. Zootaxa 1846: 1-20.

Santos, R.S. and N.M. Martinelli. 2009a. Primeiro registro de Fidicinoides picea (Walker, 1850) e Fidicinoides poulaini Boulard and Martinelli, 1996 no Brasil. Ciência Rural, Santa Maria 39: 559-562.

Santos, R.S. and N.M. Martinellli. 2009b. Descrição de novas espécies de Fidicinoides Boulard and Martinelli (Hemiptera: Cicadidae) do Brasil. Neotropical Entomology 38: 638-642.

Santos, R.S., N.M. Martinelli, D.H.B. Maccagnan, A.F. Sanborn and R. Ribeiro. 2010. Description of a new cicada species associated with the coffee plant and an identification key to the species of Fidicinoides (Hemiptera: Cicadidae) from Brazil. Zootaxa 2602: 48-56.

Sueur, J. 2002. Cicada acoustic communication: potential sound partitioning in a multispecies community from Mexico (Hemiptera: Cicadomorpha: Cicadidae). Biological Journal of the Linnean Society 75: 379-394. 
Thouvenot, M. 2007. Une Zammara nouvelle pour la Guyane (Homoptera Cicadidae). L'Entomologiste 63: 13-14.

Wolda, H. and J.A. Ramos. 1992. Cicadas in Panama, their distribution, seasonality and diversity; p. 271-279 In D. Quintero and A. Aiello (ed.). The insects of Panama and Mesoamerica. Selected Studies. New York: Oxford University Press.
RECEIVED: March 2012

ACCEPTED: May 2012

Published ONLINE: June 2012

EDITORIAL RESPONSIBILITY: Matthew Smart 